Rev. Int. Contam. Ambie. 33 (1) 49-56, 2017

DOI: 10.20937/RICA.2017.33.01.04

\title{
EVALUACIÓN DE LA SENSIBILIDAD DEL CLADÓCERO TROPICAL Ceriodaphnia cornuta A METALES PESADOS
}

\author{
Ignacio Alejandro PÉREZ-LEGASPI ${ }^{1 *}$, Mariana GARATACHIA-VARGAS ${ }^{1}$, \\ Adriana Marisol GARCÍA-VILLAR ${ }^{1}$ e Isidoro RUBIO-FRANCHINI ${ }^{2}$
}

${ }^{1}$ División de Estudios de Posgrado e Investigación, Instituto Tecnológico de Boca del Río. Kilómetro 12, Carretera Veracruz-Córdoba, Boca del Río, Veracruz, México, C. P. 94290

${ }^{2}$ Laboratorio Estatal de Salud del estado de Aguascalientes, Instituto de Salud del Estado de Aguascalientes. Avenida Siglo XXI 105, Aguascalientes, Aguascalientes, México, C. P. 20270

*Autor para correspondencia: ialegaspi@yahoo.com

(Recibido noviembre 2015; aceptado junio 2016)

Palabras clave: Dafniidae, metal pesado, toxicidad aguda, relevancia ecológica

\section{RESUMEN}

Los cladóceros han sido utilizados como organismos de prueba para evaluar el efecto adverso de contaminantes en sistemas acuáticos. Daphnia magna y Ceriodaphnia dubia están entre los más utilizados en pruebas de toxicidad acuática. Sin embargo, son poco representativos para evaluar ecosistemas acuáticos tropicales. Ceriodaphnia cornuta es un organismo común en aguas tropicales que tolera variaciones de temperatura, distribuyéndose ampliamente en diversos humedales. En este estudio, evaluamos la sensibilidad de C. cornuta a los metales pesados: plomo, cobre, cadmio, aluminio, y cromo, a través de pruebas de toxicidad letal. Los valores nominales y reales de Concentración Letal 50 (CL50) a $48 \mathrm{~h}$ obtenidos para plomo, cadmio, aluminio, cobre y cromo fueron $(0.05$, $0.06),(0.031,0.027),(1.58,1.54),(0.02,0.02)$ y $(1.12,1.16) \mathrm{mg} / \mathrm{L}$, respectivamente. La mayor sensibilidad de este cladócero ocurrió en bajas concentraciones de cobre, cadmio y plomo, mientras que es poco sensible al aluminio. Los valores de CL50 a 48 h para C. cornuta expuesta a cobre, cadmio y plomo son menores a lo reportado con otras especies de cladóceros usadas en pruebas de toxicidad letal. Lo anterior muestra una mayor sensibilidad que $D$. magna y $C$. dubia, incluso es comparable a lo reportado para otras especies de cladóceros tropicales. En conclusión, C. cornuta puede ser considerada como un organismo de prueba adecuado para el monitoreo de ecosistemas costeros tropicales contaminados con metales como cobre, cadmio y plomo presentes en el Golfo de México.

Key words: Daphniidae, heavy metal, acute toxicity, ecological relevance

\begin{abstract}
Cladocerans have been used as test organisms to assess the adverse effects of pollutants in aquatic systems. Both Daphnia magna and Ceriodaphnia dubia are among the most commonly cladocerans used in aquatic toxicity tests. However, they are inadequate to assess tropical aquatic ecosystems. Ceriodaphnia cornuta is a common organism in tropical waters which tolerates variations in temperature, and is widely distributed
\end{abstract}


in several wetlands. In this study, we evaluated the sensitivity of $C$. cornuta to heavy metals: lead, copper, cadmium, aluminum, and chromium by means of lethal toxicity tests. The Lethal Concentration 50 (LC50) at $48 \mathrm{~h}$, both nominal and real values for lead, cadmiun, copper and chromium are $(0.05,0.06),(0.031,0.027),(1.58,1.54)$, $(0.02,0.02)$ and $(1.12,1.16) \mathrm{mg} / \mathrm{L}$, respectively. This cladoceran was highly sensitive to low concentrations of copper, cadmium and lead, although it was not very sensitive to aluminum. The LC50 $48 \mathrm{~h}$ values for $C$. cornuta exposed to copper, cadmium, and lead are lower than those LC50 reported with other cladoceran species used in lethal toxicity tests, showing greater sensitivity than D. magna and C. dubia, even being comparable to that reported for other tropical cladoceran species. In conclusion, $C$. cornuta might be considered as a test organism, suitable for the monitoring of tropical coastal ecosystems contaminated with metals such as copper, cadmium and lead present in the Gulf of Mexico.

\section{INTRODUCCIÓN}

En los sedimentos de los principales sistemas lagunares costeros del Golfo de México se ha reportado la presencia de metales pesados y compuestos orgánicos (De la Maza y Bernardez 2004). Algunos de estos contaminantes como plomo, cadmio, cromo y níquel han sido registrados en altas concentraciones para estos sistemas acuáticos (Vázquez-Botello et al. 2004). La toxicidad provocada por metales en organismos acuáticos tiene efectos letales, subletales y crónicos, lo que puede ocasionar desde una reducción ligera en la velocidad de crecimiento, hasta la muerte (Leland y Kuwabara 1985). Estos efectos adversos pueden ser evaluados mediante pruebas de toxicidad que en condiciones estandarizadas y reproducibles son muy útiles para estimar los efectos de xenobióticos en organismos vivos (Rand y Petrocelli 1985).

Los cladóceros son considerados representantes ideales del zooplancton dulceacuícola, ocupando un lugar clave en la red trófica, son conspicuos, con altas tasas de reproducción, y en laboratorio son fáciles de cultivar (Mount y Norberg 1984, Dodson y Hanazato 1995, Freitas y Rocha 2011). Incluso, son de fácil manejo, con ciclos de vida cortos y su reproducción asexual permite obtener cultivos monoclonales, con gran sensibilidad a la presencia de sustancias contaminantes, lo que son ventajas durante su implementación en pruebas de toxicidad (Freitas y Rocha 2011). La mayoría de los estudios con cladóceros comprenden pruebas de toxicidad letal o aguda como parámetro de evaluación, sin embargo, es posible evaluar el crecimiento, reproducción, supervivencia y demás alteraciones fisiológicas (Villarroel 2004). Entre las especies de cladóceros más utilizadas en ensayos de toxicología acuática están Daphnia magna y Ceriodaphnia dubia (Dodson y Hanazato 1995, Martínez-Jerónimo y Rico-Martínez 2009, Freitas y
Rocha 2011). Estas especies son avaladas por diversas agencias internacionales como: la de Protección Ambiental de Estados Unidos (USEPA, por sus siglas en inglés), Sociedad Americana para la Evaluación y Materiales (ASTM, por sus siglas en inglés), Asociación Pública Americana para la Salud (APHA, por sus siglas en inglés), así como por la Norma Mexicana NMX-AA-087-SCFI-2010 (2011). Sin embargo, ambas especies son de climas fríos y templados, por tanto no pueden ser consideradas representativas para el monitoreo de ecosistemas acuáticos tropicales. Por otro lado, la Norma Mexicana NMX-AA-110-1995SCFI (1996), avala el uso del crustáceo de la misma clase (Branchiopoda) Artemia franciscana como una alternativa para monitorear sistemas costeros salobres y marinos mediante la toxicidad aguda.

En el Golfo de México, no se ha establecido un programa de monitoreo para evaluar la presencia de sustancias contaminantes en sistemas acuáticos costeros (Gold 2004). Incluso, no está considerado el uso de invertebrados como organismos de prueba. Por tanto, es necesario desarrollar, incrementar y proponer el uso de organismos de prueba representativos de ecosistemas tropicales. Lo anterior debido a que son sensibles a la presencia de sustancias contaminantes en sistemas acuáticos así, se podría implementar su uso como herramientas para el monitoreo de la calidad del agua en lagunas costeras, ya que por sus características representan el mejor índice de calidad de la cuenca. Existen pocas investigaciones con cladóceros tropicales como Daphnia exilis (Martínez-Jerónimo et al. 2008), D. similis (Rodgher et al. 2010), Pseudosida ramosa (Freitas y Rocha 2011) y Ceriodaphnia cornuta (Do Hong et al. 2004, Hong y Li 2007, Gusso-Choueri et al. 2012). Esta última especie se considera uno de los tres cladóceros más abundantes en los cuerpos de aguas tropicales perteneciente a la Familia Dafniidae (Villalobos y González 2006). Por tanto, es conveniente 
evaluar la sensibilidad a sustancias contaminantes con C. cornuta en ecosistemas costeros tropicales con la finalidad de contribuir al establecimiento de los límites máximos permisibles de sistemas acuáticos receptores de descargas residuales. El objetivo de este estudio comprendió evaluar la sensibilidad del cladócero $C$. cornuta a seis metales pesados mediante pruebas de toxicidad letal en condiciones de laboratorio, comparando los resultados con otros cladóceros y Artemia, así como su viabilidad para evaluar metales pesados en lagunas costeras tropicales del Golfo de México.

\section{MATERIALES Y MÉTODOS}

El cladócero tropical Ceriodaphnia cornuta fue colectado del sistema lagunar de Alvarado, municipio de Alvarado (18 53' - $18^{\circ} 25^{\prime}$ latitud Norte y 95 $34^{\prime}$ longitud oeste), al suroccidente del Golfo de México y sureste del estado de Veracruz. La región tiene una temperatura promedio de $18-26^{\circ} \mathrm{C}$ (De la Lanza-Espino y Lozano-Montes 1999, Pérez-Legaspi et al. 2015).

Se mantuvieron cultivos monoclonales de $C$. cornuta por más de tres años en medio dulceacuícola artificial de la Agencia de Protección Ambiental de Estados Unidos (USEPA, por sus siglas en inglés; $96 \mathrm{mg} / \mathrm{L} \mathrm{NaHCO} 3,60 \mathrm{mg} / \mathrm{L} \mathrm{CaSO}_{4} \cdot 2 \mathrm{H}_{2} \mathrm{O}, 60 \mathrm{mg} / \mathrm{L}$ $\mathrm{MgSO}_{4}$, y $4 \mathrm{mg} / \mathrm{L} \mathrm{KCl}$ ) preparado en agua deionizada con un $\mathrm{pH}$ entre $7.4-7.8$ (USEPA 1985), a una temperatura de $25 \pm 2{ }^{\circ} \mathrm{C}$ en iluminación continua, alimentando a los organismos con las microalgas Selenastrum capricornutm (Pseudokirchneriella subcapitata) y Scenedesmus obliquus (1:1) cultivadas en medio basal Bold (Nichols 1973) de acuerdo con el protocolo de Pérez-Legaspi et al. (2015).

Las pruebas de toxicidad se realizaron con ligeras modificaciones según los criterios de aceptabilidad para pruebas de toxicidad aguda con Ceriodaphnia dubia para efluentes y aguas receptoras (USEPA 2002). Los metales pesados utilizados procedieron de estándares de absorción atómica de la más alta pureza disponible (J.T. Baker, Sigma Co. EUA). Para estimar las concentraciones y parámetros reales usados en este estudio, se analizó la concentración más baja de cada solución madre para plomo, cromo, cadmio y aluminio, por horno de grafito, mientras que el cobre fue analizado por flama. El instrumento utilizado fue el espectrofotómetro de absorción atómica AAnalyst 800 Perkin-Elmer, las lámparas usadas fueron de cátodo hueco, a excepción del plomo, que fue de descarga sin electrodo. Se realizaron las correspondientes curvas de calibración en el rango lineal para cada elemento con un coeficiente de determinación
$\left(\mathrm{R}^{2}\right)$ de 0.995 así como un porcentaje de varianza entre réplicas inferior al $5 \%$.

\section{Diseño experimental}

La estandarización de las pruebas de toxicidad letal consideró como punto de partida la búsqueda de los rangos de exposición, incluyendo cinco concentraciones nominales del tóxico a evaluar y un testigo negativo (medio USEPA sin tóxico), con una mortalidad menor al $10 \%$ para el testigo negativo. Por otro lado, para cada tóxico se consideraron series logarítmicas: una concentración mínima donde no se registró mortalidad de los individuos expuestos, una concentración máxima con el $100 \%$ de mortalidad y tres concentraciones intermedias para validar así, las pruebas de toxicidad letal. Las concentraciones nominales a evaluar por metal fueron: plomo $(0.01,0.05,0.1,0.5,1.0 \mathrm{mg} / \mathrm{L})$, cobre $(0.001,0.005,0.01,0.05,0.1 \mathrm{mg} / \mathrm{L})$, cadmio $(0.001,0.005,0.01,0.025,0.05 \mathrm{mg} / \mathrm{L})$, aluminio $(0.5,1.0,1.5,2.0,3.0 \mathrm{mg} / \mathrm{L})$ y cromo $(0.1,1.0,1.5$, $2.0,3.0 \mathrm{mg} / \mathrm{L})$. Los bioensayos comenzaron con la selección de suficientes hembras de $C$. cornuta con huevos partenogenéticos en su cámara embrionaria. Se colocaron con una pipeta de transferencia y un microscopio estereoscópico en una caja Petri con medio USEPA sin alimento a $25 \pm 2{ }^{\circ} \mathrm{C}$. Posteriormente, se obtuvieron cladóceros recién nacidos (neonatos $<24 \mathrm{~h}$ ), se utilizaron para las pruebas de toxicidad, solamente neonatos a partir de la tercera generación. En las pruebas definitivas se colocaron 10 neonatos por poza en una placa nueva y estéril de 24 pozas de poliestireno (Costar Co.). Se consideró un testigo negativo y cinco concentraciones nominales del metal a evaluar, con lo que se obtuvo un volumen final de prueba de $2 \mathrm{~mL}$ por poza. Se expusieron a los cladóceros a los metales por $48 \mathrm{~h}$, sin alimento. Las placas fueron colocadas en una incubadora (ARSA) en la oscuridad a $25 \pm$ $2{ }^{\circ} \mathrm{C}$. Estas pruebas de toxicidad incluyeron tres réplicas para cada tratamiento, con tres repeticiones. Al término del periodo de exposición se registró el número de individuos muertos o inmóviles por cada tratamiento, se aceptó una mortalidad menor al $10 \%$ para el testigo negativo.

\section{Análisis estadístico}

La obtención de los valores de CL50 (concentración letal donde el $50 \%$ de los individuos expuestos muere), fue a través del análisis de datos mediante el método Probit con el programa $\mathrm{DL}_{50}$ (S.B.I.-I.R.C.T. Montpellier, Fevrier, 1987), así como pruebas de $\mathrm{X}^{2}$ para validar el ajuste de la linealidad $(\mathrm{p}<0.05)$. 
También se realizaron los siguientes análisis estadísticos con el paquete STATISTICA 7.0 StatSoft, Inc. (1984-2004): a) regresión lineal simple para estimar el coeficiente de determinación $\left(\mathrm{R}^{2}\right)$ entre las variables evaluadas, $\mathrm{b}$ ) análisis de varianza de un factor (ANDEVA) y c) pruebas de comparación de Tukey para todos los tratamientos $(\mathrm{p}<0.05)$. Se obtuvieron valores de concentración donde no se observa un efecto significativo respecto al testigo (CNOE), y de concentración mínima donde se observa un efecto significativo respecto al testigo (CMOE), respectivamente $(\mathrm{p}<0.05)$. Los valores de estos parámetros se transformaron a concentraciones reales, de acuerdo con los resultados de las concentraciones más bajas por espectrofotometría de absorción atómica. También se calculó la proporción entre valores reales y nominales, según Pérez-Legaspi et al. (2012).

\section{RESULTADOS}

La determinación analítica de la concentración nominal más baja de cada metal evaluado, mostró las siguientes concentraciones reales: plomo $0.01169 \mathrm{mg} / \mathrm{L}$, cobre $0.00101 \mathrm{mg} / \mathrm{L}$, cadmio $0.000949 \mathrm{mg} / \mathrm{L}$, aluminio $0.494 \mathrm{mg} / \mathrm{L}$, y cromo $0.102 \mathrm{mg} / \mathrm{L}$. Los rangos de toxicidad definitivos para evaluar el efecto de cada metal en el cladócero C. cornuta, fueron las siguientes concentraciones reales: plomo $(0.01,0.06,0.1,0.6$, $1.3 \mathrm{mg} / \mathrm{L})$, cobre $(0.001,0.005,0.01,0.05,0.1 \mathrm{mg} / \mathrm{L})$, cadmio $(0.0009,0.004,0.009,0.022,0.045 \mathrm{mg} / \mathrm{L})$, aluminio $(0.48,0.97,1.46,1.95,2.92 \mathrm{mg} / \mathrm{L})$ y cromo $(0.1,1.0,1.5,2.0,3.1 \mathrm{mg} / \mathrm{L})$. Los valores estimados al comparar las concentraciones nominales con las reales para CL50, CNOE, y CMOE obtenidos a partir de las pruebas de toxicidad se muestran en el cuadro I. De acuerdo con los valores reales de CL50 a 48 h, las pruebas indican que Ceriodaphnia cornuta es más sensible a los metales pesados en el siguiente orden: cobre, cadmio y plomo. Mientras que para el cromo y aluminio su sensibilidad fue menor. Del mismo modo, los valores de CMOE muestran que los metales cobre, plomo y cadmio son aquellos que tienen mayor efecto adverso a concentraciones de $0.05,0.06$, y $0.45 \mathrm{mg} / \mathrm{L}$, respectivamente. Además, los valores de $\mathrm{CNOE}$, muestran que este cladócero tiene mayor tolerancia al aluminio $(0.97 \mathrm{mg} / \mathrm{L})$.

\section{DISCUSIÓN}

El cladócero tropical C. cornuta usado como organismo de prueba en este estudio, indica mayor sensibilidad a los metales pesados evaluados en comparación con otros cladóceros de prueba y $A r-$ temia. Los valores reales de CL50 a $48 \mathrm{~h}$ registrados en el cuadro I, muestran que la sensibilidad de este organismo es mayor al cobre $(\mathrm{CL} 50=0.021 \mathrm{mg} / \mathrm{L})$, cadmio $($ CL50 $=0.027 \mathrm{mg} / \mathrm{L})$ y plomo $($ CL50 $=$ $0.068 \mathrm{mg} / \mathrm{L})$. Por otro lado, la menor sensibilidad fue registrada para cromo (CL50 $=1.16 \mathrm{mg} / \mathrm{L})$ y aluminio $(\mathrm{CL} 50=1.54 \mathrm{mg} / \mathrm{L})$. Las concentraciones

CUADRO I. VALORES DE TOXICIDAD LETALA $48 \mathrm{~h}$ OBTENIDOS PARA Ceriodaphnia cornuta EXPUESTA A DIFERENTES METALES PESADOS

\begin{tabular}{lccccc}
\hline Parámetro & Cobre & Cadmio & Plomo & Cromo & Aluminio \\
\hline CL50 (N) & 0.021 & 0.031 & 0.05 & 1.12 & 1.58 \\
CL50 (R) & 0.021 & 0.027 & 0.068 & 1.16 & 1.54 \\
Proporción & 0.98 & 1.11 & 0.73 & 0.96 & 1.02 \\
CNOE (N) & 0.01 & 0.01 & 0.01 & 0.1 & 1.0 \\
CNOE (R) & 0.01 & 0.009 & 0.013 & 0.1 & 0.97 \\
Proporción & 1.00 & 1.11 & 0.76 & 0.96 & 1.02 \\
CMOE (N) & 0.05 & 0.25 & 0.05 & 1.0 & 1.5 \\
CMOE (R) & 0.05 & 0.045 & 0.068 & 1.0 & 1.46 \\
Proporción & 0.98 & 1.11 & 0.73 & 0.96 & 1.02 \\
LC (N) & $(0.010-0.047)$ & $(0.015-0.062)$ & $(0.027-0.092)$ & $(0.703-1.799)$ & $(1.087-2.295)$ \\
LC (R) & $(0.010-0.047)$ & $(0.013-0.055)$ & $(0.036-0.125)$ & $(0.732-1.871)$ & $(1.061-2.240)$ \\
CV (\%) & 92.30 & 66.98 & 71.32 & 87.28 & 83.93 \\
R $^{2}$ & 0.9644 & 0.5638 & 0.6791 & 0.7667 & 0.9607
\end{tabular}

Abreviaturas: CL50 = concentración letal donde el $50 \%$ de los individuos expuestos muere. LC = límites de confianza del 95\% para los valores de CL50. CNOE = concentración donde no se observa un efecto significativo con respecto al testigo. $\mathrm{CMOE}=$ concentración mínima donde se observa un efecto significativo con respecto al testigo. $\mathrm{CV}=$ coeficiente de variación. $\mathrm{R}^{2}=$ coeficiente de determinación. $(\mathrm{N})=$ concentración nominal. $(\mathrm{R})$ concentración real. Proporción $=$ grado de similitud entre concentraciones reales y nominales. Valores expresados en $\mathrm{mg} / \mathrm{L}$ 
mínimas donde se observa un efecto (CMOE) previo a la muerte para $C$. cornuta se muestran en el cuadro I, en el siguiente orden de sensibilidad: cobre $<$ plomo $<$ cadmio $<$ cromo $<$ aluminio. Los valores del cobre, plomo y aluminio fueron muy cercanos al valor de CL50 a $48 \mathrm{~h}$, lo que sugiere que el efecto inmediato es la muerte. Mientras que los valores donde no se observa un efecto significativo (CNOE) están por debajo del valor CL50 a 48 h aunque son muy cercanos entre ellos. Lo que sugiere la posibilidad de que al incrementar su concentración al doble es posible afectar la supervivencia de $C$. cornuta como en la exposición al cobre. Además, la proporción obtenida entre valores reales y nominales en el cuadro I, indica que las concentraciones nominales evaluadas son muy similares a la concentración real calculada.

La comparación de valores reales de CL50 a 48 h obtenidos en este estudio para C. cornuta con lo reportado para otras especies de cladóceros expuestas a los mismos metales se muestran en el cuadro II. Se observa que $C$. cornuta es más sensible a cobre que M. macrocopa (Wong 1992), D. magna (Guilhermino et al. 2000) y D. carinata (Cooper et al. 2009). Asimismo, su sensibilidad al cadmio es mayor en comparación con D. magna (Taylor et al. 1998), D. pulex (Mount y Norberg 1984), C. dubia (Schubauer-Berigan et al. 1993) y la especie tropical D. similis (Rodhger et al. 2010). No obstante, otro cladócero tropical como P. ramosa (Freitas y Rocha 2011) es ligeramente más sensible que $C$. cornuta. La sensibilidad de $C$. cornuta expuesta a plomo es mayor que D. magna (Altindağ et al. 2008), $C$. dubia (Schubauer-Berigan et al. 1993, Cooper et al. 2009) y D. carinata (Cooper et al. 2009). Al comparar los valores CL50 a $48 \mathrm{~h}$ de cromo en $C$. cornuta, su sensibilidad es mayor que $D$. magna (Guilhermino et al. 2000). Sin embargo, aunque los otros cladóceros indican mayor sensibilidad que $C$. cornuta, es conveniente considerar que los bioensayos efectuados en este estudio fueron usando cromo a partir de $\mathrm{CrCl}_{6}$, mientras que para las otras especies se utilizaron $\mathrm{K}_{2} \mathrm{Cr}_{2} \mathrm{O}_{7}$ y/o $\mathrm{Na}_{2} \mathrm{Cr}_{2} \mathrm{O}_{7}$, lo que probablemente indique que la toxicidad se deba al cromo asociado al oxígeno. Es posible que el anión cromato sea más reactivo y en consecuencia más tóxico, mientras que el cromo usado en este estudio se disocia en cloro y cromo, siendo menos tóxico. Do Hong et al. (2004) reportaron el valor CE50 a $24 \mathrm{~h}$ para $C$. cornuta expuesta a cromo a partir de $\mathrm{K}_{2} \mathrm{Cr}_{2} \mathrm{O}_{7}$, cuyo resultado mostró una mayor sensibilidad que M. macrocopa (Wong 1992) y D. magna (Guilhermino et al. 2000, Martínez-Jerónimo et al.
2006). En el caso del aluminio, $C$. cornuta es más sensible que $D$. magna aunque el metal proviene de $\mathrm{Al}_{2} \mathrm{O}_{3}$ (Zhu et al. 2009), no obstante, los estudios que estiman el valor de CL50 en cladóceros para aluminio son escasos.

Del mismo modo, la comparación de valores reales de CL50 a 48h obtenidos para C. cornuta con lo reportado para Artemia sp., se muestran en el cuadro II. $C$. cornuta resultó ser más sensible a cobre que $A$. salina (Zulkifli et al. 2014), A. franciscana (Manfra et al. 2015) y Artemia sp. (Gajbhiye y Hirota 1990), así como más sensible a cadmio que $A$. salina (Zulkifli et al. 2014) y Artemia sp. (Gajbhiye y Hirota 1990), más sensible a plomo que Artemia sp. (Gajbhiye y Hirota 1990) y más sensible a cromo que $A$. salina (Alyuruk et al. 2013) y $A$. franciscana (Huang et al. 2015). No obstante, $A$. parthenogenetica (Nagarajan et al. 2014) muestra mayor sensibilidad a aluminio que $C$. cornuta, probablemente debido al tiempo de exposición o al $\mathrm{Al}_{2} \mathrm{Cl}_{3}$ usado para evaluar su toxicidad. Con base en lo anterior, C. cornuta es un buen candidato para evaluar sistemas costeros salobres contaminados, sin embargo es conveniente evaluar su sensibilidad a diferentes salinidades.

Este estudio demuestra que $C$. cornuta es un cladócero con sensibilidad a sustancias tóxicas comparable a otras especies de cladóceros que son avaladas por agencias internacionales y normas. Además, otros estudios de toxicidad realizados con C. cornuta reportan mayor sensibilidad que otros cladóceros y otros taxa usados como organismos de prueba (Do Hong et al. 2004, Hong y Li 2007, Gusso-Choueri et al. 2012). Esto sugiere que D. magna y $C$. dubia no son especies recomendables para monitorear ecosistemas tropicales y subtropicales, a pesar de su aclimatación, sensibilidad y aceptación por las agencias internacionales (Freitas y Rocha 2011, Gusso-Choueri et al. 2012). Por tanto, la relevancia ecológica de especies exóticas o de climas templados debe ser considerada para monitorear el impacto ambiental en sistemas acuáticos tropicales o subtropicales (Martínez-Jerónimo et al. 2008). El cladócero $C$. cornuta por su amplia distribución en estos sistemas acuáticos, ofrece gran relevancia ecológica y sensibilidad a sustancias contaminantes, lo que permite considerarlo como buen candidato para evaluar la calidad del agua. Además, por sus características biológicas, fácil manejo y alta tasa reproductiva permiten la estandarización y reproducibilidad de este organismo en bioensayos de toxicidad (Do Hong et al. 2004, Hong y Li 2007, Gusso-Choueri et al. 2012). Por lo antes mencionado, C. cornuta puede 
CUADRO II. COMPARACIÓN DE LA SENSIBILIDAD DE Ceriodaphnia cornuta CON OTROS BRANQUIÓPODOS EXPUESTOS A LOS METALES USADOS EN ESTE ESTUDIO

\begin{tabular}{|c|c|c|c|}
\hline Metal & Organismo de prueba & CL50 (mg/l) & Referencia \\
\hline Cobre & $\begin{array}{l}\text { C. cornuta } \\
\text { M. macrocopa } \\
\text { C. dubia } \\
\text { C. dubia } \\
\text { C. dubia } \\
\text { D. magna } \\
\text { D. ambigua } \\
\text { D. carinata } \\
\text { A. salina } \\
\text { A. franciscana } \\
\text { Artemia } \text { sp. }\end{array}$ & $\begin{array}{c}0.021 \\
0.08^{\mathrm{a}} \\
(0.009-0.2)^{\mathrm{b}} \\
0.0041^{\mathrm{a}} \\
0.018 \\
0.0826^{\mathrm{a}} \\
0.0065^{\mathrm{a}} \\
0.0373 \\
0.28 \\
2.51^{\mathrm{a}} \\
4.0^{\mathrm{c}}\end{array}$ & $\begin{array}{l}\text { Este estudio } \\
\text { Wong (1992) } \\
\text { Schubauer-Berigan et al. (1993) } \\
\text { Harmon et al. (2003) } \\
\text { Cooper et al. (2009) } \\
\text { Guilhermino et al. (2000) } \\
\text { Harmon et al. (2003) } \\
\text { Cooper et al. (2009) } \\
\text { Zulkifli et al. (2014) } \\
\text { Manfra et al. (2015) } \\
\text { Gajbhiye y Hirota (1990). }\end{array}$ \\
\hline Cadmio & $\begin{array}{l}\text { C. cornuta } \\
\text { D. similis } \\
\text { D. pulex } \\
\text { D. magna } \\
\text { D. magna } \\
\text { P. ramosa } \\
\text { C. dubia } \\
\text { A. salina } \\
\text { Artemia } \mathrm{sp} \text {. }\end{array}$ & $\begin{array}{l}0.027 \\
0.057^{\mathrm{d}} \\
0.068 \\
0.12 \\
0.017^{\mathrm{e}} \\
0.012^{\mathrm{e}} \\
(0.12-0.56)^{\mathrm{c}} \\
2.47 \\
1.5^{\mathrm{e}}\end{array}$ & $\begin{array}{l}\text { Este estudio } \\
\text { Rodhger et al. (2010) } \\
\text { Mount y Norberg (1984) } \\
\text { Taylor et al. (1998) } \\
\text { Guilhermino et al. (2000) } \\
\text { Freitas y Rocha (2011) } \\
\text { Schubauer-Berigan et al. (1993) } \\
\text { Zulkifli et al. (2014) } \\
\text { Gajbhiye y Hirota (1990). }\end{array}$ \\
\hline Plomo & $\begin{array}{l}\text { C. cornuta } \\
\text { C. dubia } \\
\text { C. dubia } \\
\text { D. carinata } \\
\text { D. magna } \\
\text { Artemia } \text { sp. }\end{array}$ & $\begin{array}{c}0.068 \\
(0.28-2.7)^{\mathrm{f}} \\
0.208 \\
0.444 \\
0.441^{\mathrm{g}}(24 \mathrm{~h}) \\
1.4^{\mathrm{f}}\end{array}$ & $\begin{array}{l}\text { Este estudio } \\
\text { Schubauer-Berigan et al. (1993) } \\
\text { Cooper et al. (2009) } \\
\text { “، ، } \\
\text { Altindağ et al. (2008) } \\
\text { Gajbhiye y Hirota (1990). }\end{array}$ \\
\hline Cromo & $\begin{array}{l}\text { C. cornuta } \\
\text { C. cornuta } \\
\text { M. macrocopa } \\
\text { D. similis } \\
\text { D. exilis } \\
\text { D. magna } \\
\text { D. magna } \\
\text { D. magna } \\
\text { D. magna } \\
\text { D. pulex } \\
\text { P. ramosa } \\
\text { Artemia salina } \\
\text { Artemia franciscana }\end{array}$ & $\begin{array}{l}1.16 \\
0.27^{\mathrm{h}}(24 \mathrm{~h}) \\
0.36^{\mathrm{h}} \\
0.027^{\mathrm{h}} \\
0.082^{\mathrm{h}} \\
0.022^{-154^{\mathrm{h}}} \\
0.778^{\mathrm{i}} \\
21.53^{\mathrm{j}} \\
(0.024-0.17) \\
0.029^{\mathrm{h}} \\
70^{\mathrm{h}} \\
32^{\mathrm{h}}\end{array}$ & $\begin{array}{l}\text { Este estudio } \\
\text { Do Hong et al. (2004) } \\
\text { Wong (1992) } \\
\text { Rodhger et al. (2010) } \\
\text { Martínez-Jerónimo et al. (2008) } \\
\text { Mount y Norberg (1984) } \\
\text { Martínez-Jerónimo et al. (2006) } \\
\text { Guilhermino et al. (2000) } \\
\text { “ “ } \\
\text { Dorn et al. (1987) } \\
\text { Freitas y Rocha (2011) } \\
\text { Alyuruk et al. (2013). } \\
\text { Huang et al. (2015). }\end{array}$ \\
\hline Aluminio & $\begin{array}{l}\text { C. cornuta } \\
\text { D. magna } \\
\text { A. parthenogenetica }\end{array}$ & $\begin{array}{c}1.54 \\
162.39^{\mathrm{k}} \\
0.0144^{1}(96 \mathrm{~h})\end{array}$ & $\begin{array}{l}\text { Este estudio } \\
\text { Zhu et al. (2009) } \\
\text { Nagarajan et al. (2014) }\end{array}$ \\
\hline
\end{tabular}

ser considerada una alternativa viable para sustituir a D. magna y C. dubia como especies de prueba durante el monitoreo de zonas costeras tropicales y subtropicales contaminadas con metales pesados y otras sustancias contaminantes.

\section{CONCLUSIONES}

El cladócero C. cornuta puede considerarse un buen candidato como organismo de prueba en bioensayos de toxicidad. Lo anterior por sus características biológicas, 
relevancia ecológica y gran sensibilidad a los metales pesados evaluados en este estudio en comparación con otros cladóceros como D. magna y C. dubia. Lo anterior permite considerar su uso para el monitoreo de la calidad de agua de ecosistemas costeros contaminados con los metales pesados como cobre, cadmio, plomo, cromo y aluminio, presentes en las lagunas costeras tropicales y subtropicales del Golfo de México.

\section{REFERENCIAS}

Altindağ A., Ergönül M. B., Yigit S. y Baykan Ö. (2008). The acute toxicity of lead nitrate on Daphnia magna Straus. Afr. J. Biotechnol. 7, 23, 4298-4300.

DOI: $10.5897 / A J B 08.635$

Alyuruk H., Demir G. K. y Cavas L. (2013). A video tracking based improvement of acute toxicity test on Artemia salina. Mar. Freshw. Behav. Physiol. 46, 251266. DOI: $10.1080 / 10236244.2013 .814224$

Cooper N. L., Bidwell J. R. y Kumar A. (2009). Toxicity of copper, lead, and zinc mixtures to Ceriodaphnia dubia and Daphnia carinata. Ecotox. Environ. Safe. 72, 1523-1528. DOI: 10.1016/j.ecoenv.2009.03.002

De la Lanza-Espino G. y Lozano-Montes H. (1999). Comparación fisicoquímica de las lagunas de Alvarado y Términos. Hidrobiológica 9, 15-30.

De la Maza R. y Bernardez A. (2004). Perspectivas de la conservación en el Golfo de México. En: Diagnóstico ambiental del Golfo de México. Vol. 2. (M. Caso, I. Pisanty y E. Ezcurra, Eds.). Instituto Nacional de Ecología-Secretaría de Medio Ambiente y Recursos Naturales (INE-SEMARNAT), Harte Research Institute for Gulf of Mexico Studies. Ciudad de México, México, pp. 637-656.

Do Hong L. CH., Becker-Van Slooten K. y Tarradellas J. (2004). Tropical ecotoxicity testing with Ceriodaphnia Cornuta. Environ. Toxicol. 19, 497-504.

DOI: $10.1002 /$ tox.20055

Dodson S. I. y Hanazato T. (1995). Commentary on effects of anthropogenic and natural organic chemicals on development, swimming behavior, and reproduction of Daphnia, a key member of aquatic ecosystems. Environ. Health Perspect. 103, 4, 7-11.

Dorn P. B., Rodgers J. H. Jr., Jop K. M., Raia J. C. y Dickson K. L. (1987). Hexavalent chromium as a reference toxicant in effluent toxicity tests. Environ. Toxicol. Chem. 6, 435-444. DOI: 10.1002/etc.5620060604

Freitas E. y Rocha O. (2011). Acute toxicity tests with the tropical cladoceran Pseudosida ramosa: The importance of using native species as test organisms. Arch. Environ. Contam. Toxicol. 60, 241-249.

DOI: $10.1007 / \mathrm{s} 00244-010-9541-2$
Gajbhiye S. N. y Hirota R. (1990). Toxicity of heavy metals to brine shrimp Artemia. J. Indian Fish. Assoc. 20, 43-50.

Gold G. (2004). Hidrocarburos en el sur del Golfo de México. En: Diagnóstico ambiental del Golfo de México. Vol. 2. (M. Caso, I. Pisanty y E. Ezcurra, Eds.). Instituto Nacional de Ecología-Secretaría de Medio Ambiente y Recursos Naturales (INE-SEMARNAT), Harte Research Institute for Gulf of Mexico Studies. Ciudad de México, México, pp. 657-682.

Guilhermino L., Diamantino T., Silva M. C. y Soares A. M. V. M. (2000). Acute toxicity test with Daphnia magna: An alternative to mammals in the prescreening of chemical toxicity? Ecotox. Environ. Safe. 46, 357-362. DOI: 10.1006/eesa.2000.1916

Gusso-Choueri P. K., Choueri R. B., Lombardi A. T. y Melão M. G. G. (2012). Effects of dietary copper on life-history traits of a tropical freshwater cladoceran. Arch. Environ. Contam. Toxicol. 62, 589-598.

DOI: $10.1007 / \mathrm{s} 00244-011-9725-4$

Harmon S. M., Specht W. L. y Chandler G. T. (2003). A comparison of the Daphnids Ceriodaphnia dubia and Daphnia ambigua for their utilization in routine toxicity testing in the Southeastern Unites States. Arch. Environ. Contam. Toxicol. 45, 79-85.

DOI: $10.1007 / \mathrm{s} 00244-002-0116-8$

Hong L. y Li M. H. (2007). Acute toxicity of 4-Nonylphenol to aquatic invertebrates in Taiwan. Bull. Environ. Contam. Toxicol. 78: 445-449.

DOI: $10.1007 / \mathrm{s} 00128-007-9216-5$

Huang Y., Aldasoro C. C. R., Persoone G. y Wlodkowic D. (2015). Integrated microfluidic technology for sublethal and behavioral marine ecotoxicity biotest. Memorias. SPIE -The International Society for Optical Engineering 9518. Mayo 2015. DOI: 10.1117/12.2180692

Leland H. V. y Kuwabara J. S. (1985). Trace metals. En: Fundamentals of aquatic toxicology. (G. M. Rand y S. R. Petrocelli, Eds.). Hemisphere. Washington, EUA. pp. 374-404.

Manfra L., Canepa S., Piazza V. y Faimali M. (2016). Lethal and sublethal endpoints observed for Artemia exposed to two reference toxicants and an ecotoxicological concern organic compound. Ecotox. Environ. Safe. 123, 60-64. DOI: 10.1016/j.ecoenv.2015.08.017

Martínez-Jerónimo F., Martínez-Jerónimo L. y EspinosaChávez F. (2006). Effect of culture conditions and mother's age on the sensitivity of Daphnia magna Straus 1820 (Cladocera) neonates to hexavalent chromium. Ecotoxicology 15, 259-266. DOI: $10.1007 / \mathrm{s} 10646-006-0057-5$

Martínez-Jerónimo F., Rodríguez-Estrada J. y MartínezJerónimo L. (2008). Daphnia exilis Herrick, 1895 (Crustacea: Cladocera). Una especie zooplanctónica 
potencialmente utilizable como organismo de prueba en bioensayos de toxicidad aguda en ambientes tropicales y subtropicales. Rev. Int. Contam. Ambie. 24, 153-159.

Martínez-Jerónimo F. y Rico-Martínez R. (2009). Ecotoxicología acuática. En: Toxicología Ambiental. (F. Jaramillo-Juárez, A. R. Rincón-Sánchez y R. RicoMartínez, Eds.). Universidad Autónoma de Aguascalientes - Universidad de Guadalajara. México, pp. 173-198.

Mount D. I. y Norberg T. J. (1984). A seven life-cycle cladoceran toxicity test. Environ. Toxicol. Chem. 3, 425-434. DOI: 10.1002/etc.5620030307

Nagarajan S., Sasikala S.I., Kulandaivel S. y Prakash R. (2014). Toxic effects of $\mathrm{AlCl} 3$ on Biochemical profile and fecundity of brine shrimp (Artemia parthenogenetica). Int. J. Curr. Microbiol. Appl. Sci. 3, 12, 268-275.

Nichols H. W. (1973). Growth media - freshwater. En: Handbook of phycological methods. (J. R. Stein, Ed.). Cambridge University Press. Londres, Inglaterra, pp. 7-24.

NMX-AA-110-1995-SCFI. (1996). Análisis de agua - Evaluación de toxicidad aguda con Artemia franciscana Kellog (Crustacea - Anostraca) - Método de prueba. Secretaría de Comercio y Fomento Industrial. Diario Oficial de la Federación. 12 de abril de 1996.

NMX-AA-087-SCFI-2010. (2010). Análisis de agua Evaluación de toxicidad aguda con Daphnia magna, Straus (Crustacea - Cladocera) - Método de prueba. Secretaría de Comercio y Fomento Industrial. Diario Oficial de la Federación. 3 de marzo del 2011.

Pérez-Legaspi I. A., Quintanar J. L. y Rico-Martínez R. (2012). Comparing toxicity endpoints on Lecane quadridentata (Rotifera: Monogononta) exposed to two anticholinesterases pesticides. Environ. Toxicol. 27, 518-525. DOI: $10.1002 /$ tox.20668

Pérez-Legaspi I. A., García-Villar A. M., GaratachiaVargas M., Hernández-Vergara M. P., Pérez-Rostro C. I. y Ortega-Clemente L. A. (2015). Influencia de la temperatura y tipo de alimento en la historia de vida de Ceriodaphnia cornuta SARS 1885 (Crustacea: Cladocera). Revista Investigación y Ciencia de la Universidad Autónoma de Aguascalientes 64, 11-18.

Rand G. M. y Petrocelli S. R. (1985). Fundamentals of aquatic toxicology. Hemisphere. Washington, EUA, $666 \mathrm{pp}$.

Rodgher S., Gaeta E. L. y Lombardi A. T. (2010). Suitability of Daphnia similis as an alternative organism in ecotoxicological tests: implications for metal toxicity. Ecotoxicology 19, 1027-1033.

DOI: $10.1007 / \mathrm{s} 10646-010-0484-1$
Schubauer-Berigan M. K., Dierkes J. P., Monson P. D. y Ankley G. T. (1993). pH-Dependent toxicity of Cd, $\mathrm{Cu}, \mathrm{Ni}, \mathrm{Pb}$ and $\mathrm{Zn}$ to Ceriodaphnia dubia, Pimephales promelas, Hyallela azteca and Lumbricus variegatus. Environ. Toxicol. Chem. 2, 1261-1266.

DOI: $10.1002 /$ etc. 5620120715

Taylor G., Baird D. J. y Soares A. M. V. M. (1998). Surface binding contaminants by algae: consequences for lethal toxicity and feeding to Daphnia magna Straus. Environ. Toxicol. Chem. 17, 412-419.

DOI: $10.1002 /$ etc. 5620170310

USEPA (1985). EPA-600/4-85-013. Methods for measuring the acute toxicity of effluents to freshwater and marine organisms. Office of Research and Development, United States Environmental Protection Agency. Cincinnati, OH, EUA, $231 \mathrm{pp}$.

USEPA (2002). EPA-821-R-02-012. Methods for measuring the acute toxicity of effluents and receiving waters to freshwater and marine organisms. Office of Water, United States Environmental Protection Agency, Washington, DC, EUA, 275 pp.

Vázquez-Botello A., Villanueva-Fragoso S. y Rosales-Hoz L. (2004). Distribución y contaminación de metales en el Golfo de México. En: Diagnóstico ambiental del Golfo de México. Vol. 2. (M. Caso, I. Pisanty y E. Ezcurra, Eds.). Instituto Nacional de Ecología-Secretaría de Medio Ambiente y Recursos Naturales (INE-SEMARNAT), Harte Research Institute for Gulf of Mexico Studies. Ciudad de México, México, pp. 683-712.

Villalobos M. y González E. J. (2006). Estudios sobre la biología y ecología de Ceriodaphnia cornuta Sars. Una revisión. Interciencia 31, 351-357.

Villarroel M. J. (2004). Alteraciones fisiológicas en el crustáceo Daphnia magna por exposición a plaguicidas. Tesis de Doctorado. Departamento de Biología Funcional y Antropología Física. Universitat de Valencia. Valencia, España, 223 pp.

Wong C. K. (1992). Effect of chromium, copper, nickel, and zinc on survival and feeding of the cladoceran Moina macrocopa. Bull. Environ. Contam. Toxicol. 49, 593-599. DOI: 10.1007/BF00196304

Zhu X., Zhu L., Chen Y. y Tian S. (2009). Acute toxicities of six manufactured nanomaterial suspensions to Daphnia magna. J. Nanopart. Res. 11, 67-75. DOI: $10.1007 / \mathrm{s} 11051-008-9426-8$

Zulkifli S.Z., Abdul F.Z., Mohd S.Z. y Ahmad I. (2014). Nauplii of brine shrimp (Artemia salina) as a potential toxicity testing organism for heavy metals contamination. En: From sources to solution. (A.Z. Aris, T.H. Tengku, R. Harun, A.M. Abdullah y M.Y. Ishak, Eds.). Springer, Singapur, pp. 233-237. 